\title{
Synthesis of Novel Thieno[3,2-b]thienobis(silolothiophene) Based Low Bandgap Polymers for Organic Photovoltaics
}

\author{
Bob C. Schroeder, ${ }^{a}$ Raja Shahid Ashraf, ${ }^{* a}$ Stuart Thomas, ${ }^{b}$ Andrew J. P. White, ${ }^{a}$ Laure Biniek, ${ }^{a}$ \\ Christian B. Nielsen, ${ }^{a}$ Weimin Zhang, ${ }^{a}$ Zhenggang Huang, ${ }^{a}$ Pabitra Shakya Tuladhar, ${ }^{a}$ Scott E. Watkins, \\ ${ }_{5}$ Thomas D. Anthopoulos, ${ }^{b}$ James R. Durrant $^{a}$ and Iain McCulloch ${ }^{a}$
}

Received (in $X X X, X X X)$ Xth $X X X X X X X X X 20 X X$, Accepted $X$ th $X X X X X X X X X 20 X X$

DOI: 10.1039/b000000x

Thieno[3,2-b]thienobis(silolothiophene), a new electron rich hexacyclic monomer has been synthesized and incorporated 10 into three novel donor-acceptor low-bandgap polymers. By carefully choosing the acceptor co-monomer, the energy levels of the polymers could be modulated and high power conversion efficiencies of $5.52 \%$ were reached in OPV devices.

15 The development of organic photovoltaic cells has received a lot of attention during the last decade and performances of $10 \%$ have now been achieved. ${ }^{1,2}$ The design of new semiconducting materials has played a crucial role in this development and recent donor-acceptor polymers have little structural similarity with the 20 well studied poly(3-hexylthiophene) (P3HT). ${ }^{3}$ These polymers must be considered as not only photon absorbers in bulk heterojunction (BHJ) solar cells, but also with regard to future commercialization, ambient stability and solution processability, which are key criteria in design. Indacenodithiophene (IDT), a

25 ladder type donor moiety has been successfully introduced into semiconducting polymers, which exhibited excellent hole mobilities in organic field effect transistors (OFET) and good photocurrent efficiencies in organic photovoltaic cells (OPV). ${ }^{4-7}$ We have demonstrated that the judicious choice of bridging atom 30 can not only have an influence on the crystallinity and solubility, but also allows modulating the frontier energy levels. ${ }^{8}$ The introduction of silicon as a bridging atom between adjacent aromatic units has several beneficial effects besides increasing the effective conjugation length and reducing the conformational 35 disorder. The tetravalent silicon offers the possibility to attach solubilising alkyl side chains, which allows tuning the solubility and processability of the polymer. Given the fact that a siliconcarbon bond is slightly longer than a carbon-carbon bond, the anti-bonding lobes located on the butadiene fragments adjacent to 40 the silicon bridge are separated further from each other, which ultimately should lead to a reduction of the highest occupied molecular orbital (HOMO) energy level. ${ }^{9}$ This reduction of the HOMO manifests as an increase in solar cell open circuit voltage and a potential increase in efficiency.

45 The substitution of the central benzene ring in the IDT unit with a less aromatic thieno[3,2- $b$ ]thiophene has been shown to promote the electron donating character of the monomer. ${ }^{10}$ Due to synthetic limitations, the hexacyclic thieno[3,2-b]thiophene based donor units reported so far are substituted with para-alkylated 50 benzene rings, which can lead to undesirable nanoscale morphologies, causing low fill factors (FF) in OPV devices and limiting performance to $2.5 \% .^{10,11}$ Herein we report a high yielding and versatile synthetic route towards a new silicon bridged ladder type donor, thieno[3,2- $b]$ thienobis 55 (silolothiophene) (Si4T). The incorporation of silicon as a bridging atom should therefore not only have an effect on the frontier energy levels, but also allow introducing linear alkyl side chains onto the hexacyclic donor moiety.

The synthesis of Si4T and the corresponding polymers is 60 shown in Scheme 1 . The $\alpha$-positions of commercially available 3,6-dibromothieno[3,2- $b$ ] thiophene (1) were iodated and via a selective Negishi coupling between 2 and (3-bromothiophen-2yl)zinc bromide, the 3,6-dibromo-2,5-bis(3-bromothiophen-2yl)thieno[3,2- $b$ ] thiophene (3) could be assembled. In order to 65 improve solubility of $\mathbf{3}$ and to minimize the possibility of sidereactions during the following synthetic steps, the free $\alpha$-positions of 3 were lithiated and subsequently quenched with chlorotrimethylsilane to afford compound $\mathbf{4}$. The ring-closure was induced by lithiation of all four brominated positions on 70 compound $\mathbf{4}$ and by slowly quenching the reaction with dichlorodioctylsilane. Single crystals of $\mathbf{5}$ were grown from an acetonitrile/diethyl ether mixture, and X-ray studies confirmed both the chemical structure and the planarity of the fused Si4T unit, the central $\mathrm{C}_{14} \mathrm{Si}_{4} \mathrm{~S}_{4}$ unit being coplanar to within ca. $0.03 \AA$ 75 (Figure 1).

In order to incorporate the new hexacyclic donor unit into lowbandgap polymers via Stille coupling, the trimethylsilane groups
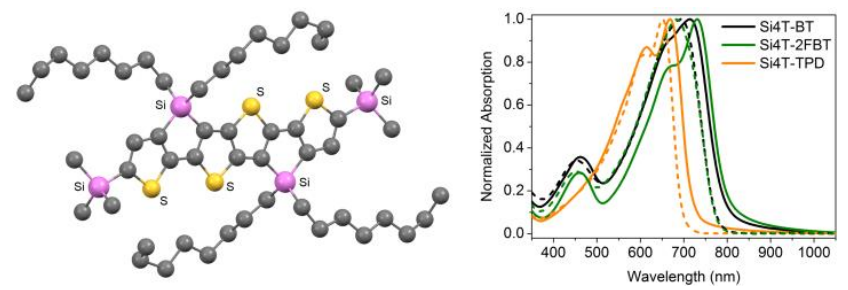

Figure 1. Single crystal structure of compound 5 (left). Normalized 80 optical absorption spectra of Si4T polymers (right) in dilute chlorobenzene (CB) solution (dashed lines) and as thin films (full lines) spin-cast from $\mathrm{CB}$ solution. 


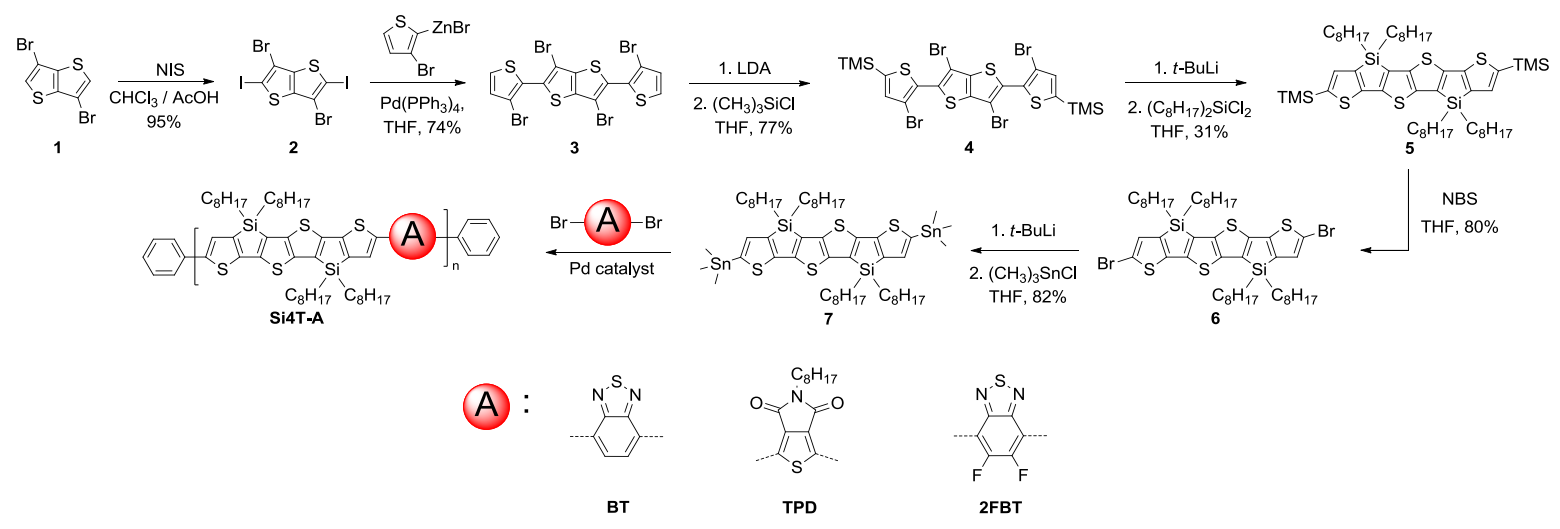

Scheme 1. Synthetic route towards the novel Si4T monomer and polymers.

were cleaved with $\mathrm{N}$-bromosuccinimide before compound 6 was

5 stannylated. All three polymers were synthesized by microwave assisted Stille polycondensations by reacting one equivalent of distannylated monomer $\mathbf{7}$ with one equivalent of the corresponding dibrominated co-monomer (A). All polymers were purified by a series of Soxhlet extractions and were recovered as 10 dark fibrous solids with high molecular weights (Table 1). Thermal gravimetric analysis (TGA) (Figure S5 in the ESI) confirmed that all Si4T polymers are stable above $420^{\circ} \mathrm{C}$. Differential scanning calorimetry (DSC) did not show significant thermal transitions for any of the polymers (Figure S6 in the ESI).

15 Density functional theoretical (DFT) studies predict the lowest lying HOMO energy level for the Si4T-TPD polymer and a homogenous distribution of both frontier energy levels along the polymer backbone (Figure S8 in the ESI). The energy levels for Si4T-BT and Si4T-2FBT are calculated to be the same, with the ${ }_{20} \mathrm{HOMO}$ in both cases delocalized over the polymer backbone, but the LUMO mainly localized on the BT and 2FBT units respectively (Figures S7 and S9 in the ESI).

Polymer Si4T-BT was obtained by co-polymerizing monomer 7 with benzo[ $c][1,2,5]$ thiadiazole $(\mathbf{B T})$. The polymer has a very 25 broad thin film absorption in the visible range extending from 400 up to $800 \mathrm{~nm}$ with two absorption bands, one with a maximum around $450 \mathrm{~nm}$ from the $\pi-\pi^{*}$ transition and a much stronger intramolecular charge transfer (ICT) absorption band with a maximum at $714 \mathrm{~nm}$ (Figure 1). The ionisation potential of 30 the polymers were measured as thin films by photoelectron spectroscopy in air (PESA). The measured HOMO energy level of Si4T-BT was around $-5.0 \mathrm{eV}$. Replacement of the BT unit with thieno[3,4-c]pyrrole-4,6-dione (TPD) led to an $0.3 \mathrm{eV}$ lower HOMO energy level in the Si4T-TPD polymer (Table 2). 35 However, given the fact that TPD is a weaker acceptor than BT, the absorption spectra of Si4T-TPD were significantly blue

Table 1. Molecular Weights and Thermal Stability of the Si4T Polymers

$\begin{array}{ccccc}\text { Polymer } & M_{\mathrm{n}}{ }^{a}(\mathrm{~kg} / \mathrm{mol}) & M_{\mathrm{w}}{ }^{b}(\mathrm{~kg} / \mathrm{mol}) & \mathrm{PDI}^{c} & T_{\mathrm{d}}{ }^{d}\left({ }^{\circ} \mathrm{C}\right) \\ \text { Si4T-BT } & 19 & 53 & 2.8 & 428 \\ \text { Si4T-TPD } & 21 & 26 & 1.2 & 423 \\ \text { Si4T-2FBT } & 40 & 58 & 1.5 & 428\end{array}$

\footnotetext{
${ }^{a}$ Number-average molecular weight. ${ }^{b}$ Weight-average molecular weight.

${ }^{c} M_{\mathrm{w}} / M_{\mathrm{n}} .{ }^{d}$ Decomposition temperature (5\% weight loss) determined by 40 thermal gravimetric analysis under nitrogen.
}

shifted, both in solution and thin film and the bandgap was raised by $0.1 \mathrm{eV}$. In thin film, the absorption band of Si4T-TPD presents a more defined vibronic structure, than in solution, with two peak maxima at $615 \mathrm{~nm}$ and at $668 \mathrm{~nm}$, respectively. In order 45 to reduce the HOMO energy level of the Si4T polymers without compromising the bandgap, monomer 7 was co-polymerized with 5,6-difluorobenzo[c][1,2,5]thiadiazole (2FBT). Due to the electron withdrawing character of fluorine, both frontier energy levels should be similarly lowered and therefore the bandgap is 50 expected to remain unaffected. ${ }^{12}$ The absorption spectrum of Si4T-2FBT in solution is nearly identical to that of Si4T-BT. In the solid state, however there are significant differences between the spectra of both polymers. The Si4T-2FBT spectrum is further red-shifted than the Si4T-BT spectrum, perhaps correlated to the 55 higher number average molecular weight of Si4T-2FBT. The Si4T-BT absorption band shows some indication of a shoulder at lower wavelengths, whereas the absorption band of Si4T-2FBT presents a well defined shoulder feature at higher energies. We speculate that the more defined vibronic structure of the Si4T${ }_{60}$ 2FBT absorption band is caused by a reduction of the rotational freedom between donor and acceptor units in the polymer backbone, due to favourable attractive interactions between the sulphur's lone pair electrons on the Si4T unit and the electronegative fluorine atoms on the adjacent BT moiety. This ${ }_{65}$ hypothesis is also supported by the quantum mechanical calculations, which suggest a dihedral angle of $3.87^{\circ}$ between the Si4T and the BT unit and a slightly smaller angle of $2.87^{\circ}$ in case of the Si4T-2FBT polymer. The barrier of planarization, defined as the difference between the energy of the planar (constraint) 70 and the fully optimized structures, of Si4T-BT amounts to 0.23

Table 2. Optical Properties and Frontier Energy Levels of Si4T Polymers

\begin{tabular}{|c|c|c|c|c|c|}
\hline Polymer & $\lambda_{\max }{ }^{a}(\mathrm{~nm})$ & $\lambda_{\max }^{b}(\mathrm{~nm})$ & $\begin{array}{l}\text { exp. HOMO / } \\
\text { LUMO }^{c}(\mathrm{eV})\end{array}$ & $\begin{array}{l}\text { calc. HOMO / } \\
\mathrm{LUMO}^{d}(\mathrm{eV})\end{array}$ & $E_{\mathrm{g}}{ }^{e}$ \\
\hline Si4T-BT & 694 & 714 & $-5.0 /-3.4$ & $-4.5 /-3.1$ & 1.6 \\
\hline Si4T-TPD & 651 & 668 & $-5.3 /-3.6$ & $-4.7 /-2.9$ & 1.7 \\
\hline Si4T-2FBT & 684 & 731 & $-5.1 /-3.5$ & $-4.6 /-3.1$ & 1.6 \\
\hline
\end{tabular}

${ }^{a}$ Dilute CB solution. ${ }^{b}$ Thin film spin-coated from CB solution $(5 \mathrm{mg} / \mathrm{ml}$, $1000 \mathrm{rpm}){ }^{c}$ LUMO energy level estimated by adding the absorption onset to the HOMO energy level which was measured by photoelectron 75 spectroscopy in air. ${ }^{d}$ energy levels calculated by DFT using B3LYP/6$31 \mathrm{G}^{*}$ model. ${ }^{e}$ Optical band gap determined from the onset of absorption in the solid state. 

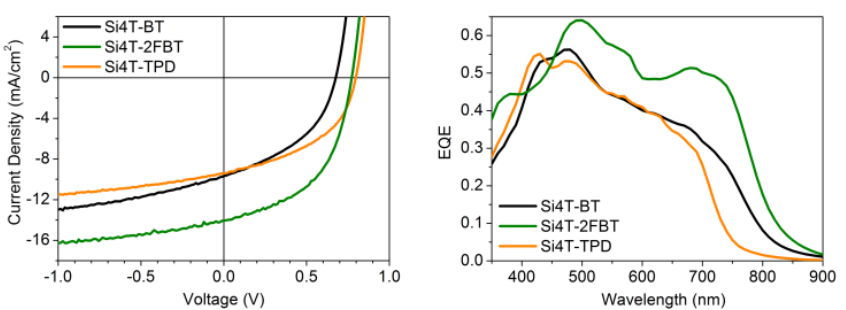

Figure 2. J-V curve (left) and external quantum efficiencies spectra (right) of Si4T-polymer:PC ${ }_{71} \mathrm{BM}$ OPV devices.

$\mathrm{kJ} / \mathrm{mol}$, which is nearly double the energy needed to planarize Si4T-2FBT $(0.12 \mathrm{~kJ} / \mathrm{mol})$.

5 The HOMO energy levels of Si4T-BT and Si4T-2FBT are both higher than the corresponding silaindacenodithiophene (SiIDT) polymers previously reported. ${ }^{13}, 14$ The increase in HOMO energy level confirms that the incorporation of the more electron rich thieno[3,2-b]thiophene into the IDT unit 10 significantly increases the electron rich character of the fused ring system. The absorption spectra of Si4T polymers are about 60-80 $\mathrm{nm}$ red shifted compared to SiIDT polymers. When blended with [6,6]-phenyl C71 butyric acid methyl ester $\left(\mathrm{PC}_{71} \mathrm{BM}\right)$, Si4T-BT afforded a $V_{O C}$ of $0.67 \mathrm{~V}$ and a short-circuit current $\left(J_{S C}\right)$ of 9.67 $15 \mathrm{~mA} / \mathrm{cm}^{2}$ in OPV devices, which corresponds to a rather low photocurrent efficiency (PCE) of $2.79 \%$ (Table 3). The Si4TTPD polymer has a much lower HOMO energy level, which translates into a higher $V_{O C}$ of $0.80 \mathrm{~V}$ (Figure 3). However, a similar $J_{S C}$ value of $9.42 \mathrm{~mA} / \mathrm{cm}^{2}$ and the moderate FF of 0.46 20 resulted in average device performances of $3.46 \%$. The Si4T2FBT polymer exhibited the best performance in OPV devices. The low HOMO energy level of the Si4T-2FBT polymer manifests as a large $V_{O C}$ of $0.77 \mathrm{~V}$ comparable to the Si4T-TPD polymer, but the $J_{S C}$ increased by nearly $70 \%$ to $14.07 \mathrm{~mA} / \mathrm{cm}^{2}$ 25 which led to high device efficiencies of $5.52 \%$. The external quantum efficiency (EQE) spectra are shown in Figure 2 and all three devices exhibit a broad response from 350 up to $750 \mathrm{~nm}$ for Si4T-TPD, up to $800 \mathrm{~nm}$ in the cases of Si4T-BT and Si4T2FBT, respectively. However, the lower EQE response at higher 30 wavelengths suggests that in the case of Si4T-BT and Si4T-TPD most of the current is generated by the $\mathrm{PC}_{71} \mathrm{BM}$ and not by polymer excitons. Si4T-2FBT is the only of the three polymers with an EQE above $40 \%$ between 600 and $750 \mathrm{~nm}$, which therefore contributes significantly to the charge generation in the ${ }_{35}$ OPV device at higher wavelengths.

The surface morphologies of the polymer: $\mathrm{PC}_{71} \mathrm{BM}$ blends were studied by atomic force microscopy (Figures S10 to S12 in the ESI). All blends form very smooth high quality films when spincasted from $o$-dichlorobenzene. The three films are very 40 homogenous with little apparent phase separation and low surface roughness. The Si4T-2FBT film is slightly rougher than the other

Table 3. Photovoltaic Properties of the Si4T Polymers

$\begin{array}{ccccc}\text { Polymer } & J_{S C}\left(\mathrm{~mA} / \mathrm{cm}^{2}\right) & V_{O C}(\mathrm{~V}) & \text { FF } & \text { PCE }(\%) \\ \text { Si4T-BT } & 9.69 & 0.67 & 0.43 & 2.79 \\ \text { Si4T-TPD } & 9.42 & 0.80 & 0.46 & 3.46 \\ \text { Si4T-2FBT } & 14.07 & 0.77 & 0.51 & 5.52\end{array}$

Conventional device architecture ITO/PEDOT:PSS/polymer:PC ${ }_{71} \mathrm{BM} /$ $\mathrm{Ca} / \mathrm{Al}$ with a polymer:PC $\mathrm{PC}_{71} \mathrm{BM}$ blend ratio of 1:3.5 processed from $o$ 45 dichlorobenzene. films and contains small granular features. The formation of these granular domains might be favoured by the higher molecular weight component of the polymer, which tends to preferentially favour phase separation from the $\mathrm{PC}_{71} \mathrm{BM}$.

50 In conclusion, we have synthesized a novel thieno[3,2$b$ ] thiophene based ladder type donor monomer, whose fused and planar structure was determined by single crystal X-ray diffraction. The incorporation of silicon bridging atoms allowed the introduction of linear alkyl side chains onto the novel 55 thieno[3,2-b]thienobis(silolothiophene) monomer and we synthesized three soluble and easily processable new donoracceptor polymers. By judiciously choosing the acceptor monomer, we were able to adjust the polymer frontier energy levels, which lead to high $V_{O C}$ values and excellent PCE values of ${ }_{60} 5.52 \%$ in OPV devices.

\section{Notes and references}

${ }^{a}$ Department of Chemistry and Centre for Plastic Electronics, Imperial College London, London, SW7 2AZ, UK; E-mail:

r.ashraf@imperial.ac.uk

${ }_{65}{ }^{b}$ Department of Physics and Centre for Plastic Electronics, Imperial College London, London, SW7 2AZ, UK

${ }^{c}$ CSIRO Materials Science and Engineering, VIC 3169, Australia

$\dagger$ Electronic Supplementary Information (ESI) available: Experimental details, characterization data, AFM images, graphical representations of 70 frontier energy levels and crystallographic data. See DOI: $10.1039 / \mathrm{b} 000000 \mathrm{x} /$

1. G. Dennler, M. C. Scharber, T. Ameri, P. Denk, K. Forberich, C Waldauf and C. J. Brabec, Advanced Materials, 2008, 20, 579-583.

2. J. Peet, A. J. Heeger and G. C. Bazan, Accounts of Chemical 75 Research, 2009, 42, 1700-1708.

3. C. L. Chochos and S. A. Choulis, Progress in Polymer Science, 2011, 36, 1326-1414.

4. C. Yu, C. Chen, S. Chan, G. Hwang, C. Ting, Chemistry of Materials, 2009, 21, 3262-3269.

80 5. W. M. Zhang, J. Smith, S. E. Watkins, R. Gysel, M. McGehee, A. Salleo, J. Kirkpatrick, S. Ashraf, T. Anthopoulos, M. Heeney and I. McCulloch, Journal of the American Chemical Society, 2010, 132, 11437-11439.

6. H. Bronstein, D. S. Leem, R. Hamilton, P. Woebkenberg, S. King, 85 W. M. Zhang, R. S. Ashraf, M. Heeney, T. D. Anthopoulos, J. de Mello and I. McCulloch, Macromolecules, 2011, 44, 6649-6652.

7. J. Wang, S. K. Hau, H. Yip, J. A. Davies, K. Chen, Y. Zhang, Y. Sun and A. K.-Y. Jen, Chemistry of Materials, 2011, 23, 765-767.

8. I. McCulloch, R. S. Ashraf, L. Biniek, H. Bronstein, C. Combe, J. E. 90 Donaghey, D. I. James, C. B. Nielsen, B. C. Schroeder and W. Zhang, Accounts of Chemical Research, 2012, 45, 714-722.

9. J. Ohshita, Macromolecular Chemistry and Physics, 2009, 210, 13601370.

10. H. Bronstein, R. S. Ashraf, Y. J. Kim, A. J. P. White, T 95 Anthopoulos, K. Song, D. James, W. M. Zhang and I. McCulloch, Macromolecular Rapid Communications, 2011, 32, 1664-1668.

11. Y.-J. Cheng, C.-H. Chen, T.-Y. Lin and C.-S. Hsu, Chemistry - An Asian Journal, 2012, 7, 818-825.

12. H. Zhou, L. Yang, A. C. Stuart, S. C. Price, S. Liu and W. You, Angewandte Chemie International Edition, 2011, 50, 2995-2998.

13. R. S. Ashraf, Z. Y. Chen, D. S. Leem, H. Bronstein, W. M. Zhang, B. Schroeder, Y. Geerts, J. Smith, S. Watkins, T. D. Anthopoulos, H. Sirringhaus, J. C. de Mello, M. Heeney and I. McCulloch, Chemistry of Materials, 2011, 23, 768-770.

105 14. B. C. Schroeder, Z. Huang, R. S. Ashraf, J. Smith, P. D'Angelo, S. E. Watkins, T. D. Anthopoulos, J. R. Durrant and I. McCulloch, Advanced Functional Materials, 2012, 22, 1663-1670. 